\title{
Assessment of the bovine tuberculosis elimination protocol in the United States
}

\author{
Leslie J. Verteramo Chiu, ${ }^{1 *}$ Loren W. Tauer, ${ }^{2}$ Rebecca L. Smith, ${ }^{3}$ and Yrjo T. Grohn ${ }^{1}$ \\ ${ }^{1}$ Section of Epidemiology, College of Veterinary Medicine, Cornell University, Ithaca, NY 14853 \\ ${ }^{2}$ Charles H. Dyson School of Applied Economics and Management, Cornell S. C. Johnson College of Business, Cornell University, Ithaca, \\ NY 14853 \\ ${ }^{3}$ Department of Pathobiology, College of Veterinary Medicine, University of Illinois, Urbana 61802
}

\section{ABSTRACT}

In this study, we analyzed the performance of the USDA's bovine tuberculosis (bTB) elimination protocol in a 1,000-cow closed dairy herd using an agent-based simulation model under different levels of initial bTB infection. We followed the bTB test sensitivity and specificity values used by the USDA in its model assessment. We estimated the net present value over a 20 -yr horizon for a bTB-free milking herd and for bTB-infected herds following the USDA protocol. We estimated the expected time to identify the infection in the herd once it is introduced, its elimination time, the reproductive number $\left(R_{0}\right)$, and effective reproduction number $\left(R_{\mathrm{e}}\right)$ under the USDA protocol. The optimal number of consecutive negative whole-herd tests (WHT) needed to declare a herd bTB-free with a $95 \%$ confidence under different bTB prevalence levels was derived. Our results support the minimum number of consecutive negative WHT required by the USDA protocol to declare a herd bTB-free; however, the number of consecutive negative WHT needed to eliminate bTB in a herd depends on the sensitivity and specificity of the tests. The robustness of the protocol was analyzed under conservative bTB test parameters from the literature. The cost of implementing the USDA protocol when 1 infected heifer is introduced in a 1,000-cow dairy herd is about $\$ 1,523,161$. The average time until detection and the time required to eliminate bTB-infected animals from the herd, after 1 occult animal is introduced in the herd, were 735 and $119 \mathrm{~d}$, respectively.

Key words: agent-based model, bovine tuberculosis infection simulation, bovine tuberculosis economic cost, bovine tuberculosis elimination strategy

Received April 28, 2018.

Accepted September 7, 2018.

*Corresponding author: ljv9@cornell.edu

\section{INTRODUCTION}

Bovine tuberculosis (bTB) is a chronic disease of cattle caused by infection with the bacteria Mycobacterium bovis. The pathogen can be spread from infected cattle to other warm-blooded animals and humans through aerosol discharges, including saliva, urine, and manure contact; through feeding and watering sites; and by consuming unpasteurized milk or colostrum from infected animals. Animals infected with bTB are hard to identify from clinical symptoms alone. In the early stages of infection, bTB-infected animals do not show any clinical signs. In later stages of infection, infected animals may show weight loss, weakness, pneumonia, chronic moist cough, and enlargement of lymph nodes. Infected cows also show decrease in milk production.

Herds infected with bTB are very rare in the United States. Since the beginning of the Bovine Eradication Program in 1917, the prevalence of bTB in the United States has decreased from $5 \%$ to less than $0.006 \%$ in 2011 (Naugle at al., 2014). Despite this low prevalence rate, current costs associated with bTB control are estimated to be about $\$ 38$ million per year, or about $\$ 342$ million between 2001 and 2009, with most of the money spent in depopulating and indemnifying affected farms (USDA-APHIS, 2009). There are 3 levels of bTB classifications for a state or areas within a state: Accredited (free of bTB), Modified Accredited Advance, and Modified Accredited. An area comprising 4 counties in Michigan is the only Modified Accredited area currently in the United States (USDA-APHIS, 2017); all other states are currently classified as accreditedfree.

Herds in areas that are not accredited-free face restrictions in cattle movement. Herds not known to be affected can move out of areas that are not accreditedfree with tuberculosis testing. Cattle movement for quarantined, positive herds is restricted to slaughterhouses. In general, milk-producing farms in quarantine are not negatively affected in their daily operations; however, they will not be able to sell breeding stock, 
limiting their business opportunities. Animals younger than $12 \mathrm{mo}$ in quarantined, positive herds may be moved to approved feedlots as long as they have passed a caudal fold tuberculin (CFT) test within $60 \mathrm{~d}$. Milk originating from bTB-positive herds can be sold for human consumption, but not from bTB-positive animals in the herd. Pasteurization kills $M$. bovis and other pathogens, making the milk safe to consume. Animals that need to be culled can be sent to a slaughterhouse but are inspected at slaughter.

The surveillance and control mechanism currently in effect by the USDA begins primarily by identifying infected cattle through postmortem surveillance at the slaughterhouse, or through annual testing for some herds in non-accredited-free areas, then conducting an investigation to identify the herd of origin, apply quarantine, perform a herd test in the possible herd of origin, confirm infection in the herd, and then apply the bTB elimination protocol. Postmortem surveillance is conducted on all adult cattle at slaughterhouses by federal meat inspectors of the USDA's Food Safety and Inspection Service (FSIS). If an infected herd is detected and quarantined, it is subject to either depopulation or a test-and-cull protocol to regain bTB-free status. The costs of depopulation have been analyzed to be greater than the cost of test and cull unless the impact on the state's bTB-free status is sufficiently high (Smith at al., 2013b). Norby et al. (2004), however, concluded that total depopulation is preferred over the test-andcull strategy because of low test specificity. The current USDA bTB elimination protocol no longer recommends whole-herd depopulation as the first choice to manage bTB within a herd. Our analysis focuses on the costs associated with the option of test and cull under the USDA protocol.

The general guidelines of the USDA protocol for bTB elimination in a herd were modified in 2009 and the modified protocol usually requires less time for a herd to regain its quarantine-free status compared with the pre-2009 protocol. For comparison, the pre-2009 bTB elimination protocol was as follows. Once an infected animal was detected postmortem and the originated herd was identified, the herd was placed in quarantine and the elimination protocol began by conducting a whole-herd test (WHT) with CFT. For the first 2 WHT, all CFT-positive animals were required to be necropsied with enhanced inspection with samples for culture taken. For the following WHT, animals positive by CFT were then retested with the comparative cervical tuberculin (CCT) test. Animals that were CCT-positive were culled immediately and tested with an enhanced postmortem test to corroborate the CCT test results. The results from the enhanced postmortem test were used in declaring the bTB infectious status of the animal. The protocol required the entire herd to have 8 consecutive negative WHT to release the quarantine. The first 4 tests were performed at intervals of at least $60 \mathrm{~d}$ and at least $180 \mathrm{~d}$ was required between the fourth and fifth tests. For the sixth through eighth tests, at least 12 mo was required between consecutive tests (USDA-APHIS, 2005). This previous protocol was determined to be cost inefficient by Smith et al. (2013b, 2014).

In contrast, the current bTB elimination protocol is divided into 3 test phases: removal, verification, and assurance testing. Once an infected animal is detected postmortem, the herd is quarantined and a whole-herd test on animals, generally older than $1 \mathrm{yr}$, is applied to estimate bTB prevalence and determine the number of consecutive negative WHT at the removal phase. This initial WHT, also called test 0 , consists on a CFT, CCT, histology, and culture test evaluated in series. The protocol begins if one animal is confirmed in the culture test. The protocol begins with removal tests by testing all animals 2 mo of age and older with the CFT test. After 2 consecutive negative WHT, or more if the bTB incidence is deemed high, and $95 \%$ probability of freedom from disease, verification testing begins. The verification test is conducted at least $180 \mathrm{~d}$ after the last removal test. The verification test consists of a CFT test followed by another approved test, usually $\mathrm{CCT}$, interpreted in series. If an animal tests positive with a CFT and positive with the secondary test, it is classified as positive; otherwise, the test result is considered negative. Only animals over 6 mo of age are tested during verification testing. The quarantine is lifted if all animals test negative in verification testing. The final testing phase, assurance testing, is conducted after $1 \mathrm{yr}$ of the quarantine release. The long interval between the quarantine release and assurance testing allows a bTB infection in an undetectable animal to become detectable. Assurance testing, like verification testing, requires 2 tests interpreted in series: CFT and another approved test (CCT). It is recommended that animals 12 mo of age and older are tested in this phase. The assurance testing ends after 3 to 5 negative WHT are performed. The WHT in the assurance testing are performed annually or biennially, but the final assurance test must be conducted at most 6 yr after quarantine release. The elimination protocol starts over from the beginning (removal phase) if any animal is confirmed positive by postmortem test at any WHT.

The current protocol requires a minimum of 2 consecutive negative WHT in the removal phase and $95 \%$ confidence of freedom from disease. The probability of eliminating bTB in a herd based on initial bTB 
prevalence and number of consecutive negative WHT is analyzed in this paper.

The previous USDA bTB elimination protocol (pre2009) has been analyzed to be suboptimal in favor of shorter intervals and fewer consecutive negative WHT to declare a herd bTB-free (Smith at al., 2013b, 2014). Smith et al. (2013b) concluded that the optimal testand-cull control strategy for a bTB outbreak is a 2-mo testing interval and 2 consecutive negative WHT to declare a herd free of bTB, which is in accordance with the current protocol. Smith et al. (2013b) do not include assurance testing in their model. The authors stated that their findings are not consistent with the policies of many other high-income countries. They suggest that concern of a recurrent outbreak may explain what appears to be an overly conservative elimination protocol. They noted that the prevalence of bTB in the herd is important in determining the extended testing in the protocol. In comparison, in Australia, fewer than 4 negative WHT may be sufficient to declare a herd bTB-free because of the low prevalence rate (Radunz, 2006). More stringent testing protocols are required for higher bTB herd prevalence (Ryan et al., 2006). However, high bTB prevalence within a herd may be caused by an endemically infected wildlife population that is in contact with the cattle (Livingstone et al., 2006).

Consequently, in this study, we estimated the costs associated with the policy of testing and culling a bTB outbreak in a commercial dairy herd with a standard herd structure for the United States, following the current USDA protocol. The analysis considered 3 levels of bTB prevalence in a closed herd, assuming no wildlife infection (Dressler et al., 2010). We compared, for all initial introductions of bTB-infected animals, the costs of implementing the bTB elimination protocol as well as the time to detect and eliminate bTB in the herd, effective reproduction number, time to declare a herd bTB-free, and number of negative WHT to declare a herd bTB-free. Unlike Smith et al. (2013a,b), who used a compartment mathematical model, our analysis used an agent-based model with the individual cows as the agent. Our agent-based model is a modified version of that developed by Al-Mamun et al. (2016). Our model allows tracking of whether a cow is infected or not and the time of infection, and more accurately models the identification and culling of individual cows. Our findings are consistent with Smith at al. (2013b, 2014) and with the current USDA protocol, in which shorter test intervals and fewer negative WHT are sufficient to declare a herd bTB-free. We find that about $95 \%$ of the time, bTB-infected animals can be eliminated from the herd after 2 consecutive negative WHT.

\section{MATERIALS AND METHODS}

We developed an agent-based simulation model for a 1,000-cow dairy operation in a closed herd, with the following assumptions. All cow replacements were produced within the herd. The dairy herd was parameterized to a typical US commercial herd. The net present value (NPV) of the herd was estimated for $20 \mathrm{yr}$ of simulation with an added terminal value. It was assumed that bTB was introduced in the herd at d 1 of the simulation from infected, but unreactive, heifers. Even in a closed herd, heifers may, at times, be purchased from the market. In this analysis, we assumed that this occurred at the beginning of the simulation. After infection was introduced, no other outside animals were allowed into the herd. The number of unreactive heifers introduced in the herd were 1,5 , and 10. The current USDA protocol is tested following the USDA's bTB test sensitivities and specificities as used in their protocol evaluation. As a sensitivity analysis, we compared the USDA's model to more conservative bTB test sensitivity and specificity values from the literature, and we generated another set of results with the bTB transmission rate parameter increased and decreased by $20 \%$ from our baseline. These results are presented in the Sensitivity Analysis section. The NPV of a healthy herd was also estimated. Each simulation consisted of 1,000 iterations.

\section{Population Dynamics}

The basic population dynamics follow the model developed by Verteramo Chiu et al. (2018). Individual animals move within 3 age groups: calves $(0-60 \mathrm{~d}$ of age), heifers (61-719 d of age), and cows ( $\geq 720 \mathrm{~d}$ of age). Once a calf is born, she spends the first day of life with her dam in the nursery, and then she is transferred to the calf group, where she is kept until $60 \mathrm{~d}$ of age. Once a calf reaches $61 \mathrm{~d}$ of age, she is transferred to the heifer group. An animal is kept in the heifer group until she reaches $720 \mathrm{~d}$ of age, and at that time, she is transferred to the cow group. All heifers are inseminated at $440 \mathrm{~d}$ of age and are expected to calve at $720 \mathrm{~d}$ of age (280-d pregnancy). Actual calving time depends on the pregnancy rate, which was stochastically modeled as in Verteramo Chiu et al. (2018).

Natural culling is determined daily for each animal and depends on the age group and parity of the animal. The natural culling rate includes involuntary and voluntary culling rates. Involuntary culling refers to culling exogenous to the farmer (e.g., accidents, natural death), whereas voluntary culling is based on the 
farmer's decision (e.g., cull a cow with mastitis or other disease). Natural culling rates and pregnancy rates for all animals and age groups are shown in Supplemental Table S1 (https://doi.org/10.3168/jds.2018-14990) and are based on Mitchell et al. (2008) with some modifications to make the herd demography stable. Natural culling rate for calves, which measures expected survival rate, was set at $4 \%$. This value is based on Silva del Río et al. (2007). Natural culling rates for cows increase with parity. Some voluntary culling decisions are not included in the natural culling rate but are accounted for in the simulation; for instance, culling cows after 8 consecutive unsuccessful inseminations or when the number of cows exceeds the herd limit. The culling rate of cows was about $36 \%$. The limit on the number of cows in the herd was set to 1,000. Excess cows were culled based on their expected milk production (cows with lower expected milk production were culled first).

\section{Infection Dynamics}

The population and infection process are illustrated in Figure 1. The subscripts in Figure 1 (1, 2, and 3) indicate the age group of the animals, where $1=$ calves; $2=$ heifers; and $3=$ cows. The bTB infection groups were designated susceptible (S): animals free from $M$. bovis; occult $(\mathrm{O})$ : animals infected at low levels but that do not show signs of infection and cannot infect other animals; reactive $(\mathrm{R})$ : animals infected and detectable by test but noninfectious; or infectious (I): infected, detectable by test, and can infect other animals. In total, there were 12 mutually exclusive groups according to age and infection level of the animal.

When a female calf is born, it spends a day with its dam, where it can become infected with probability $\lambda_{3}$. If infected, the bTB infection status of the calf becomes occult and may progress to reactive and infectious

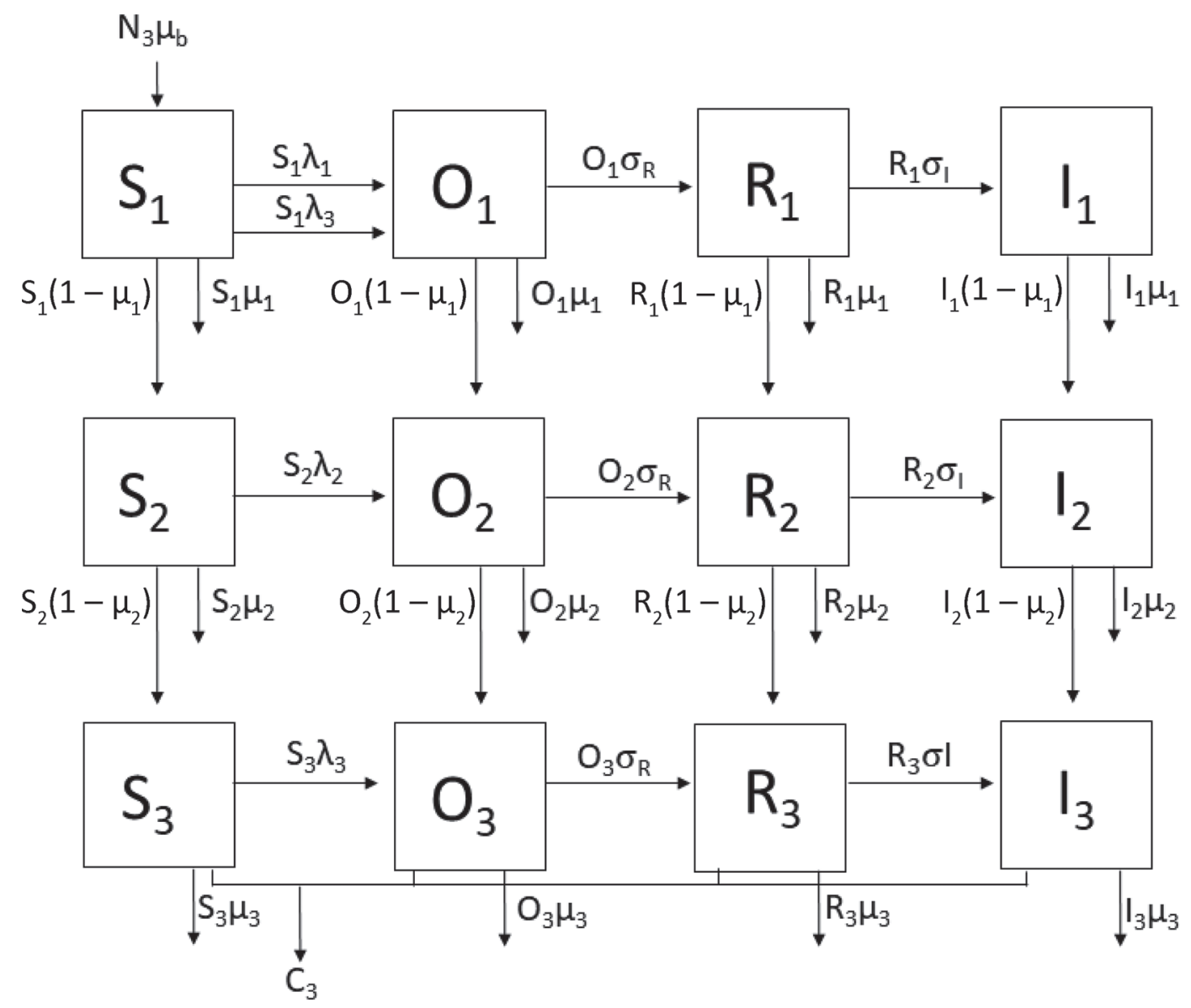

Figure 1. Animal dynamics across Mycobacterium bovis (bovine tuberculosis, bTB) infection status. There are 12 mutually exclusive animal groups based on age and infection status. Subscripts 1, 2, and 3 indicate the age group: calves, heifers, and cows, respectively. Infection status is denoted by $\mathrm{S}$ (susceptible), $\mathrm{O}$ (occult), $\mathrm{R}$ (reactive), and I (infectious). Parameters are described in Table 1. 
Table 1. Bovine tuberculosis (bTB) infection and herd parameters ${ }^{1}$

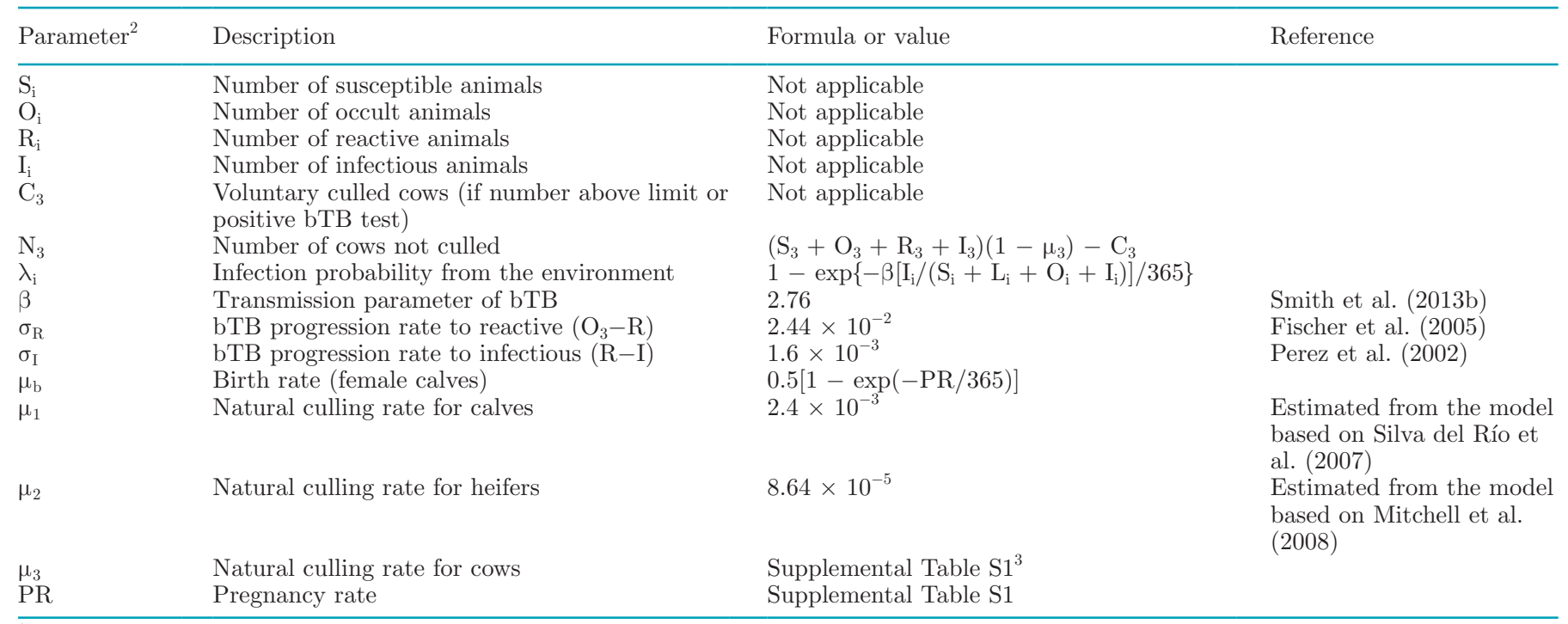

\footnotetext{
${ }^{1}$ Daily values.

${ }^{2}$ Subscript i refers to age groups, where subscripts 1,2 , and $3=$ calves, heifers, and cows, respectively.

${ }^{3}$ Supplemental Table S1; https://doi.org/10.3168/jds.2018-14990.
}

with probabilities $\sigma_{\mathrm{R}}$ and $\sigma_{\mathrm{I}}$, respectively. We assumed that the milk fed to calves is pasteurized and does not represent a transmission path. The parameter of the probability of infection is frequency dependent, and its value depends on the proportion of infected cows in the herd. Unlike density-dependent parameters, values of which depend on animal density in a compartment, the density of animals is assumed constant in a commercial dairy operation. Male newborn calves are immediately sold. After the first day of life, a female calf is transferred to the calf group and kept there until $60 \mathrm{~d}$ of age. At $61 \mathrm{~d}$ of age, all calves are transferred to the heifer group and remain there until $720 \mathrm{~d}$ of age, when they are moved to the cow group. Susceptible calves become susceptible heifers, and then become susceptible cows; this progression is similar for each infection status. Calves may become infected from the time they spent with their dam in the cow group, and from any infectious calves in the calf group with probability $\lambda_{1}$.

Susceptible heifers may become occult heifers with probability $\lambda_{2}$, and progress to reactive and infectious status with probabilities $\sigma_{\mathrm{R}}$ and $\sigma_{\mathrm{I}}$, respectively. Heifers transferred to the cow group keep their infection status. A susceptible cow is at risk of becoming an occult cow by contact with infected cows with probability $\lambda_{3}$. Similar to the other age groups, occult animals progress to the reactive stage with probability $\sigma_{R}$, and reactive animals become infectious with probability $\sigma_{\mathrm{I}}$. On average, an animal spends $43 \mathrm{~d}$ in the occult stage and 21 mo in the reactive stage.
The probability of infection for all age groups is estimated using the frequency-dependent transmission parameter, $\boldsymbol{\beta}$, with a value of 2.76 (Barlow et al., 1997). The transmission parameter and progression probability values are conservative compared with other estimates in the literature. Other studies find higher transmission parameters, but their range is very wide. For a literature review on bTB transmission parameter estimates, see Álvarez et al. (2014). Other estimates of $\beta$ range from 0.95 to 3.6 (Fischer et al., 2005) and 2.2 (Perez et al., 2002). Estimates of $\sigma_{\mathrm{R}}$ include $9.6 \times$ $10^{-4}$ (Kao et al., 1997), whereas estimates of $\sigma_{\mathrm{I}}$ include $2.3 \times 10^{-2}$ (Kao et al., 1997), and $3.5 \times 10^{-2}$ and $3.6 \times$ $10^{-3}$ (Conlan et al., 2012), for assumed and estimated values, respectively. Infection, progression, and herd parameter values are shown in Table 1.

Reactive and infectious animals can be detected through a CFT, CCT, or a postmortem test of lesions. Both CFT and CCT tests lack perfect sensitivity and specificity. For this reason, susceptible and occult animals can falsely test positive, whereas reactive and infective animals can falsely test negative.

We did not consider reproductive performance or milk yield losses of reactive or infectious bTB cows because we assumed that cows would be detected before milk reduction occurs. According to Mellado et al. (2015), reactive bTB cows show only marginal decreases in both reproductive and milk yield performance, which may explain why producers are unwilling to cull bTB reactive animals. 


\section{Costs and Revenues}

All costs and revenues of the herd operation were computed daily. The daily costs of raising a replacement heifer from newborn, as well as daily fixed costs per cow and fixed costs of calving, were obtained from Karszes (2014). Daily feed costs, measured in kilograms of DM, were obtained from the USDA's Economic Research Service (USDA-ERS, 2015). The amount of DMI depends mainly on BW, lactation, and pregnancy status. Animal BW is mainly a function of age, lactation, and pregnancy. It is estimated following the functions of Korver et al. (1985), as described in the Nutrient Requirements of Dairy Cattle (NRC, 2001).

The milk price used in the analysis was the 2015 national average as reported by the USDA's National Agricultural Statistics Service (USDA-NASS, 2017). Feed cost and milk price were measured in dollars per kilogram of milk and kept fixed for the duration of the analysis. Insemination cost and pregnancy diagnosis costs were taken from Kaniyamattam et al. (2016). Male calves were assumed sold for $\$ 150$ immediately after birth. The revenue from culling a cow is taken from the National Agricultural Statistics Service (USDA-NASS, 2017). Cost and revenue parameters and their values are shown in Table 2. The herd's NPV was estimated over 7,300 d, and no inflationary effect was assumed.

\section{bTB Elimination Strategies and Infection Scenarios}

To compare the costs associated with performing an elimination protocol of testing and culling in a dairy herd with a bTB outbreak, we estimated the NPV of a healthy herd (with no bTB infection) over $20 \mathrm{yr}$, as well as the NPV of an infected herd implementing the USDA protocol following the USDA's test parameters (sensitivity and specificity), when 1, 5, and $10 \mathrm{bTB}$ occult heifers are introduced into the herd.
Under the USDA policy, if an animal is detected with lesions resembling bTB infection during a postmortem test and confirmed with histology or culture tests, the suspected animal is traced back to its herd of origin. Once the herd is identified, it is immediately quarantined and if a bTB-positive animal is confirmed in the initial WHT (test 0), the bTB elimination protocol begins as soon as possible. There are 2 possible bTB elimination actions: depopulation and test and cull. The USDA protocol does not recommend depopulation (USDA-APHIS-VS, 2010). In this analysis, we do not analyze the costs of depopulation, but it has been shown that it is more expensive than testing and culling (Smith et al., 2013b, 2014).

When the USDA bTB test-and-cull elimination protocol is applied, the herd goes through a series of WHT at different time intervals. At the removal phase, a number of WHT are conducted until 2 consecutive negative WHT are reached and it is estimated by the USDA bTB model that there is $95 \%$ confidence of freedom from disease. The USDA estimates that herds with low initial bTB prevalence, which is the case in most of the United States, would require only 2 consecutive negative WHT to achieve that confidence level. High bTB prevalence may require more consecutive negative WHT to achieve the same confidence level. We test that assumption in this paper. The first set of tests of the protocol, called removal testing, consists of testing all animals in the herd 2 mo of age and older with CFT at intervals no shorter than $60 \mathrm{~d}$. Under some circumstances as determined by the supervising veterinarian, animals may be tested with another test. If 2 tests are used, the tests are interpreted in parallel. That is, if any of these tests is positive, the animal is considered bTB positive. In practice, the removal phase consists of only one CFT test. Including the secondary test and interpreting them in parallel increases the number of culled animals (due to increase in false positives), and

Table 2. Cost and revenue parameter description

\begin{tabular}{lcl}
\hline Parameter description & Value & Reference \\
\hline Cost per test $^{1}$ (CFT and CCT) & $\$ 7.13$ & Buhr et al. (2009) \\
Cost per test (PCR) & $\$ 271.25$ & USAHA (2004) \\
Veterinarian travel cost & $\$ 50$ & Smith et al. (2013b) \\
Maximum number of cows allowed & 1,000 & \\
Insemination cost, per event & $\$ 20$ & Kaniyamattam et al. (2016) \\
Pregnancy diagnosis cost, per event & $\$ 8$ & Kaniyamattam et al. (2016) \\
Milk price, $\$ / \mathrm{kg}$ & 0.375 & USDA-NASS (2017) \\
Dry matter intake cost, $\$ / \mathrm{kg}$ & 0.244 & USDA-ERS $(2015)$ \\
Fixed costs per cow, $\$ / \mathrm{d}$ & 2.5 & Karszes $(2014)$ \\
Discount rate, yearly & 0.05 & Assumed \\
Fixed cost of a newborn calf & $\$ 150$ & Karszes $(2014)$ \\
Male calf sale price & $\$ 150$ & Karszes (2014) \\
Culled cow price & $\$ 600$ & USDA-NASS $(2017)$ \\
\hline
\end{tabular}

${ }^{1} \mathrm{CFT}=$ caudal fold tuberculin; CCT $=$ comparative cervical tuberculin. 
costs associated with testing (Kathleen Orloski, USDAAPHIS, Fort Collins, CO; personal communication), especially in low-prevalence areas. In this study, we followed the standard practice of the USDA of administering 1 CFT test in the removal phase. Any test-positive animal is culled immediately and examined postmortem using a histology test. If the herd status is confirmed bTB positive, animals that test positive to the histology test may undergo an additional postmortem test consisting of a mycobacterial culture test. If the status of the herd is not confirmed, all bTB-suspected animals will be cultured. This culture test is considered to have a specificity of 1 . Culture test results may take between 4 and $8 \mathrm{wk}$. A WHT is considered negative if no animal tests positive to the histology or culture test (if any were performed). The WHT is considered positive if at least 1 animal tests positive in the culture test. The herd is considered bTB-free if it has 2 consecutive negative WHT and achieves a 95\% confidence level based on the USDA model. The confidence of the negative WHT results depend on the prevalence of bTB in the herd as observed in the WHT. A herd with higher prevalence may require more negative WHT than a herd with a few infected animals to reach a negative WHT of that confidence level. The number of consecutive negative WHT needed to achieve 95\% confidence based on bTB prevalence is estimated by the USDA.

Once the herd has met the requirement of the removal testing, the verification testing begins. Only animals over 6 mo of age are tested. The purpose of verification testing is to ensure that any acquired sensitivity to Mycobacterium is not related to bTB. In the verification testing, CFT and CCT tests are applied and interpreted in series, improving specificity (probability of true negative). Only CFT-positive animals are tested with CCT. The result is considered positive if both tests are positive. All positive tested animals are examined postmortem for bTB using the same postmortem tests as in the removal phase. The postmortem test determines the result of the verification test. The verification test can be applied no earlier than $180 \mathrm{~d}$ after the last removal test. If the verification test is negative, the herd is considered bTB-free and the quarantine is lifted.

Assurance testing is applied after the herd is declared bTB-free. The purpose of the assurance testing is to monitor the herd for low risks of infections that may be latent in the herd. The interval between assurance tests allows an infected but undetectable calf to be tested at an older age when it is more likely to become detectable. Assurance testing consists of 2 serial tests, like the assurance testing. First, a CFT test, and if positive, a secondary test (CCT). Any animal that tests positive to the secondary test is examined postmortem (using histology and, if positive, a culture test). The postmortem test determines the result of the assurance test. It is recommended that all animals over 12 mo of age be tested in the assurance-testing phase. Assurance testing begins $1 \mathrm{yr}$ after the quarantine is lifted. Assurance tests can be conducted annually or biennially, depending on the herd's risk of re-exposure from wildlife or introduced animals. Assurance tests are defined in a herd management plan, agreed upon by the farm manager, the state, and USDA, that specifies the testing. Assurance testing ends after 3 to 5 tests are conducted where the last test is performed 5 to $6 \mathrm{yr}$ after the quarantine release. Under the USDA protocol, a herd can regain its bTB-free status in as few as $300 \mathrm{~d}$ (USDA-APHIS-VS, n.d.). Supplemental Table S2 (https://doi.org/10.3168/ jds.2018-14990) summarizes the USDA bTB elimination protocol.

Although it is assumed that residual risk is present in a herd unless all animals exposed to an infected animal are culled (More et al., 2015), the number of assurance tests and the time between them allow susceptible animals that were infected after exposure to an infected animal enough time to become reactive to bTB tests. Some factors contributing to bTB persistence in a herd include infection from local sources, such as environment, wildlife, or introduced animals (More and Good, 2015). Conlan et al. (2012) suggest that reducing the rate of introduction into a herd is a major factor to control bTB, through better surveillance or by reducing wildlife reservoirs. Introduction of bTB into a herd through wildlife reservoirs and environment is not a significant risk factor for most US herds outside of Michigan.

We considered only the costs associated with testing and culling and not the loss of any revenue caused by the quarantine. Dairy producers are assumed to generate all their revenues from milk production and selling culled animals, and not from selling live animals to other farms, which is prohibited under quarantine. Our focus was only on the costs to eradicate bTB in a dairy herd when 1,5 , and 10 bTB-infected animals are introduced in the herd.

\section{Sensitivity and Specificity of bTB Tests}

The sensitivity of the bTB tests is critical in determining the efficacy of the elimination protocol. Sensitivity in a test is the probability that an infected animal tests positive (true positive). If sensitivity is less than 1, then the test may identify some infected animals as negative (false negative). In contrast, the test specificity is the probability that a noninfected animal tests negative. If specificity is less than 1 , then the test may wrongly identify a noninfected animal as positive (false posi- 
tive). These concepts are better explained by looking at the data layout of a test in Supplemental Table S3 (https://doi.org/10.3168/jds.2018-14990). The columns of Supplemental Table S3 represent test results, either positive $(\mathbf{T}+)$ or negative $(\mathbf{T}-)$ for a given infection. The rows represent the actual infection status of the animal, either infected $(\mathbf{I}+)$ or non-infected $(\mathbf{I}-)$. The letters $\mathrm{a}$ and $\mathrm{b}$ refer to an infected animal $(\mathrm{I}+)$ that tested positive $(\mathrm{T}+)$ and negative $(\mathrm{T}-)$, respectively. The letters $\mathrm{c}$ and $\mathrm{d}$ refer to a noninfected animal (I-) that tested positive $(\mathrm{T}+)$ and negative $(\mathrm{T}-)$, respectively. The variables $\mathrm{m}_{1}, \mathrm{~m}_{0}, \mathrm{n}_{1}, \mathrm{n}_{0}$, and $\mathrm{n}$ are the total number of infected animals (I+), noninfected animals $(\mathrm{I}-)$, test positive $(\mathrm{T}+)$, test negative $(\mathrm{T}-)$, and total number of animals being tested, respectively.

Sensitivity (Se), the probability that an infected animal tests positive, is $\operatorname{Prob}(\mathrm{T}+\mathrm{I}+)=\mathrm{a} /(\mathrm{a}+\mathrm{b})=\mathrm{a} /$ $\mathrm{m}_{1}$. Its complementary probability $(1-\mathrm{Se}), \operatorname{Prob}(\mathrm{T}-1$ $\mathrm{I}+)=\mathrm{b} /(\mathrm{a}+\mathrm{b})=\mathrm{b} / \mathrm{m}_{1}$, is the probability that an infected animal tests negative, or the probability of false negative. Specificity $(\mathbf{S p})$, the probability that a noninfected animal tests negative, is $\operatorname{Prob}(\mathrm{T}-\mid \mathrm{I}-)$ $=\mathrm{d} /(\mathrm{c}+\mathrm{d})=\mathrm{d} / \mathrm{m}_{2}$. Its complementary probability $(1-\mathrm{Sp}), \operatorname{Prob}(\mathrm{T}+\mid \mathrm{I}-)=\mathrm{d} /(\mathrm{c}+\mathrm{d})=\mathrm{d} / \mathrm{m}_{2}$, is the probability that a noninfected animal tests positive, or the probability of false positive.

In most cases, the bTB protocol uses 2 types of tests to detect bTB in an animal, CFT and CCT. These tests can be performed sequentially and the results interpreted in parallel or in series.

Because parallel interpretation is true if any of the tests are true, the sensitivity (probability of an infected animal testing positive) of the 2 tests in parallel is equal to the sum of the sensitivity of the first test and the sensitivity of the second test minus their product. That is, given events $\mathrm{A}$ and $\mathrm{B}$, the probability that either event $\mathrm{A}$ or $\mathrm{B}$ occurs is $\operatorname{Prob}(\mathrm{A}$ or $\mathrm{B})=\operatorname{Prob}(\mathrm{A})$ $+\operatorname{Prob}(\mathrm{B})-\operatorname{Prob}(\mathrm{A}$ and $\mathrm{B})$. The sensitivity of 2 tests ( $\mathrm{Se} 1, \mathrm{Se} 2)$ interpreted in parallel $(\mathrm{Sep})$ is $\mathrm{Sep}=\mathrm{Se} 1$ $+\mathrm{Se} 2-(\mathrm{Se} 1 \mathrm{Se} 2)$. The specificity of a parallel interpretation (Spp) is the product of the specificities of the tests: Spp $=$ Sp1Sp2. Similarly, the sensitivity of a series interpretation is the product of the sensitivities of the tests: Ses $=$ Se1Se2, whereas the specificity of the series interpretation is the sum of each of the tests' specificities minus their product, $\mathrm{Sps}=\mathrm{Sp} 1+\mathrm{Sp} 2-$ (Sp1Sp2). Parallel interpretation increases sensitivity and decreases specificity, leading to more animals testing positive and thus increasing costs due to extra culling and testing. These extra costs may not be justified in areas of low prevalence. For this reason, the USDA protocol follows a single CFT test in the removal phase, except in special cases as determined by the USDA.
Our simulation used the same sensitivity and specificity values as those used by the USDA to evaluate its bTB program. The test parameter values and their prior distribution are shown in Supplemental Table S4 (https://doi.org/10.3168/jds.2018-14990). Every time a test is performed in our model, a random value following its prior distribution is generated.

Other estimates of test sensitivity and specificity include the following references. In a recent paper, Nuñez-Garcia et al. (2018), using statistical meta-analyses that included logistic regression models with fixed and random effects for the UK and Ireland, estimated median CFT sensitivity at 0.76 (95\% CI: $0.56-0.89)$ and 0.96 (95\% CI: 0.88-0.98) from 2 model estimates. The sensitivity of CCT was estimated at 0.63 (95\% CI: $0.40-0.84$ ), and 0.75 (95\% CI: $0.61-0.86)$ from 2 model estimates for a "severe interpretation" of the reaction. The median specificities for both tests were estimated at a value of 1 . Prevalence of bTB in the UK and Ireland are estimated at 3.27 and $4.37 \%$ (EFSA, 2009), respectively. Using Bayesian latent class analysis, Lahuerta-Marin et al. (2018) estimated the mean CCT sensitivity in dairy productions in Northern Ireland to be 0.39 , with a $95 \%$ CI between 0.34 and 0.44 ; the mean specificity was estimated at 0.996 with a $95 \%$ CI between 0.995 and 0.997 . They mentioned that a CCT test could be a good initial test in herds that were not chronically infected. In a field study in Australia, CFT sensitivity and specificity under an apparent prevalence of $1.6 \%$ were estimated at 0.681 and 0.967 (Wood et al., 1992).

Estimates of test performance in the United States include Bruning-Fann et al. (2017), who estimated the CFT sensitivity and specificity at 0.868 and 0.927 , respectively. In a meta-analysis of field studies in the United States, Farnham et al. (2012) reported estimates of CFT sensitivity point estimates ranging from 0.804 to 0.930 , with a mean of 0.859 ; and CFT specificity point estimates ranging from 0.892 to 0.952 , with a mean of 0.928 . The sensitivity point estimates of series test interpretation of CFT-CCT ranged from 0.744 to 0.884 , with a mean of 0.795 , whereas its specificity point estimates ranged from 0.973 to 0.986 , with a mean of 0.979 . Two of the most recent studies in the meta-analysis of Farnham et al. (2012) include Norby (2003) and Norby et al. (2004). Norby et al. (2004) estimated the CFT sensitivity at 0.833 (95\% CI: $0.515-0.979)$, the series interpretation (CFT-CCT) sensitivity at 0.750 (95\% CI: $0.428-0.945)$, and the sensitivity for the necropsy test at 0.833 (95\% CI: $0.515-0.979)$. All values were estimated for a low bTB prevalence herd (1.1-5.5\%). In Norby (2003), the sensitivity and specificity of the series interpretation (CFT-CCT) was estimated at 
0.758 and 0.986 , respectively. In their meta-analysis, de la Rua-Domenech et al. (2006) presented estimates of sensitivity and specificity for CFT and CCT for both the United States and Europe. Their references for the United States include Whipple et al. (1995), which estimated a sensitivity value between 0.804 and 0.844 . The differences in test performance among different countries are due to differences in test techniques, tuberculin dosage, and interpretation (Schiller et al., 2011).

Estimations of postmortem inspection and tests parameters range widely. Education and training of abattoir inspectors are important factors in improving postmortem sensitivity (Schiller et al., 2011). In a recent paper, Willeberg et al. (2018), based on previous estimates, reported a median sensitivity for meat inspection at 0.55 (95\% CI: 0.095-0.871), and median specificity at 0.993 (95\% CI: 0.974-0.999), and the mean sensitivity and specificity for culture test at 0.77 and 1.00, respectively. Using a Bayesian partial-likelihood approach, O'Hare et al. (2014) estimated abattoir inspection sensitivity in the UK to be 0.67 . In a study in Ethiopian abattoirs, Asseged et al. (2004) calculated routine abattoir inspection sensitivity at 0.55 . In their study, Nuñez-Garcia et al. (2018) estimated the median sensitivity and specificity of the standard necropsy (meat inspection) at 0.71 (95\% CI: $0.37-0.92)$ and 1.00 (95\% CI: 0.99-1.00), respectively. The median estimated values for the sensitivity of necropsy using microscopic examination in their 2 models were $0.63(95 \%$ CI: $0.15-0.93)$ and 0.66 (95\% CI: 0.41-0.84); the median specificity estimate was 1.00 (95\% CI: 0.95-1.00). The median sensitivity for culture test was estimated at 0.74 (95\% CI: 0.46-0.94), and its median specificity at 1 (95\% CI: 0.98-1.00). All postmortem tests analyzed in Nuñez-Garcia et al. (2018) have a median specificity of at least 0.99. Downs et al. (2018) estimated the median histology test sensitivity between 0.63 and 0.66 , and its specificity at 1.00 . Culture methods to isolate $M$. bovis are considered the gold standard in testing bTB but require qualified technicians (Bermudez et al., 2010). Culture test performance can be affected by cross contamination and other factors; however, these events do not represent a significant problem in the US (Kathleen Orloski, USDA-APHIS, Fort Collins, CO; personal communication). Thus, a specificity of 1 is assumed for culture tests. We did not obtain culture test parameter values used by the USDA in their protocol evaluation; for the model, we used values from the literature.

The values of the USDA's test parameters are in general lower than those from the literature. Only the sensitivities of the necropsy and histology tests are significantly higher in the USDA's model compared with the literature cited in this paper. The average sensitivities from the relevant literature for necropsy and histology tests are 0.72 (Norby et al., 2004; O'Hare et al., 2014; Nuñez-Garcia et al., 2018; Willeberg et al., 2018) and 0.63 (Nuñez-Garcia et al., 2018), respectively. The average value from the relevant literature for CCT specificity is 0.91 (Norby, 2003; Norby et al., 2004; Farnham et al., 2012), which is similar to that of the USDA's model (0.92).

\section{Sensitivity Analysis}

We performed a sensitivity analysis on the bTB transmission rate and the bTB test parameters of the USDA. The bTB transmission parameter $\beta$ was modified from its base value of 2.76 by $20 \%$, resulting in values of 2.21 ( $\beta$ Low) and 3.31 ( $\beta$ High). These values were consistent with the estimates of Fischer et al. (2005) and Perez et al. (2002).

The bTB test parameters were modified by using the most conservative test parameter estimates from the literature review that are relevant to the US situation (e.g., values from developed countries were preferred over those estimated from developing countries). In general, the values used in the sensitivity analysis were more conservative than those used by the USDA protocol, except for the specificities of CFT, necropsy, and histology tests. In this case, our sensitivity model included those values from the USDA. The sensitivity analysis uses the lowest value either from the literature or from the USDA. Because the performance of the protocol depends on the tests parameters, the sensitivity analysis represents a low performance case of the protocol. A summary of the mean values of the test parameters used in our model, the sensitivity analysis model, and the average from relevant literature is shown in Supplemental Table S5 (https://doi.org/10 $.3168 /$ jds.2018-14990). When the literature values were estimated as medians, we estimated the mean values by fitting a $\beta$ distribution and calibrating it to their $95 \%$ CI.

\section{Model Simulation}

All simulations start from the same initial herd and continue over $7,300 \mathrm{~d}(20 \mathrm{yr})$. The initial herd is created by simulating for 3,000 d (to ensure a stable distribution of animals in a herd is reached) a herd populated with 1,000 cows under the following parity distribution: $45 \%$ in parity $1,30 \%$ in parity 2 , and $25 \%$ in parity 3. Age, pregnancy status, pregnant days, and days in milk of all initial cows in the model were uniformly distributed based on parity.

The analysis of the bTB elimination protocols starts by changing the bTB infection status of heifers from susceptible to occult. We assume that the bTB infec- 
Table 3. Net present value (NPV; SD in parentheses) of a 1,000-cow herd over a 20-yr period for different numbers of initial bovine tuberculosis (bTB)-infected animals following the USDA bTB elimination protocol

\begin{tabular}{lccc}
\hline Initial infected animals & $\mathrm{NPV}, \$$ & $\begin{array}{c}\text { Difference over no } \\
\text { infection case, } \$\end{array}$ & $\begin{array}{c}\text { Indemnity } \\
\text { cost, }{ }^{1} \$\end{array}$ \\
\hline 0 (no infection) & $7,133,305$ & & \\
1 infected animal & $(49,242)$ & $1,523,161$ & $1,266,635$ \\
& $5,610,143$ & $(52,498)$ & $(529,898)$ \\
5 infected animals & $4,814,117$ & $2,319,187$ & $(262,893)$ \\
10 infected animals & $(441,450)$ & $2,573,408$ & $1,942,453$ \\
& $(4,559,897$ & $(259,118)$ \\
\hline
\end{tabular}

${ }^{1}$ Indemnity cost is the payment given by the government to the dairy operation for all animals that test positive and are culled.

tion is introduced to a clean herd from outside heifers. These infected heifers are older than $540 \mathrm{~d}$, uniformly distributed between ages 541 and 719 d. Including a large number of occult heifers in the analysis allowed us to test the efficacy of the protocols in eliminating bTB infection in the herd.

The simulation model was built in Matlab (MathWorks, Natick, MA) to allow more control and flexibility in modeling the interaction among agents (animals) and in the design and analysis the elimination protocol. For each initial infection level, we estimated the NPV, number of days to detect bTB, number of WHT to eliminate infection, and number of infected animals at each WHT. The simulation code and all supporting files can be found at https:/github.com/lverteramo/ bTBSimulationModel.

\section{RESULTS AND DISCUSSION}

Five sets of results are presented in this analysis for each infection scenario: herd NPV, number of WHT required to eliminate $\mathrm{bTB}$, number of days to detect and eliminate bTB, estimated prevalence from initial test results, and the reproductive number, $R_{0}$, under the bTB elimination protocol.

\section{Net Present Value}

From Table 3, it can be seen that the NPV over a 20yr horizon of the healthy 1,000-cow commercial dairy herd is $\$ 7,133,305$. When 1 infected cow is introduced in the herd, the NPV decreases to $\$ 5,610,143$. That is, under the USDA bTB elimination protocol, 1 infected animal in a 1,000-cow herd incurs costs of $\$ 1,523,161$. This difference corresponds to $21 \%$ of the NPV of a healthy herd. The NPV when 5 and 10 infected animals are introduced in the herd are $\$ 4,814,117$ and $\$ 4,559,897$, respectively, which corresponds to 27 and $32 \%$ of the NPV of a healthy herd, respectively. The cost per initial infected cow decreases from $\$ 463,827$ to $\$ 257,340$, when 5 and 10 infected animals are introduced in the herd, respectively. The losses in NPV caused by the bTB elimination protocol may be partially offset by indemnity payments from the government. All animals testing positive are culled, and the government indemnifies the farm for those animals at fair market value. However, in a closed herd, the time to repopulate the farm may result in missing revenues from milk production.

The NPV differences between a healthy and an infected herd are due to the cost, number, and efficiency of testing. These costs depend on the correct identification of infected cows. Higher test sensitivity and specificity can decrease the costs associated with the elimination protocol by increasing the likelihood of detecting and eliminating infected animals quickly, while minimizing false-positive results. If infected cows fail to be identified earlier by the tests, then the number of WHT will increase as these cows are identified in later tests and the elimination protocol starts again. Failing to identify and cull infected cows earlier can increase the total number of WHT and thus elevate costs.

\section{Number of WHT Required to Eliminate bTB}

We tested whether the total number of WHT required to eliminate bTB in the herd differed depending on initial prevalence. The empirical cumulative distribution functions (CDF) of the total number of WHT needed to eliminate bTB from the herd for the 3 initial prevalence levels are shown in Figure 2. The number of total WHT needed to eliminate bTB in the herd is smaller when the number of initial infected animals introduced is smaller (Figure 2). If 1 infected animal is introduced in the herd, after $4 \mathrm{WHT}$, regardless of the test result, there is a $94.8 \%$ probability that bTBinfected animals are eliminated from the herd. If 5 and 10 infected animals are introduced in the herd, the probability of bTB elimination after 4 WHT is lower, at 89.9 and $81.8 \%$, respectively. After 5 WHT, 
the probability of bTB elimination in the herd is 97.7, 97.4, and $94.4 \%$ for 1,5 , and 10 initial bTB-infected animals, respectively. That is, with a $0.1 \%$ bTB initial prevalence in a 1,000-cow herd, bTB elimination can occur after 5 WHT with a $98 \%$ confidence. Only in the case of an outbreak of 1 infected animal can bTB infection be eliminated without any WHT $40.4 \%$ of the time. This is because the infected animal may be culled before it infects other animals or during the initial test to determine bTB prevalence. We estimated that for an outbreak of 1 infected animal, $7.3 \%$ of the time bTBinfected animals are eliminated without being detected at slaughter and $6.6 \%$ of the time an infected animal is detected at slaughter but no infected animal remains at the herd; thus, the initial test is negative and the protocol is not triggered. However, $26.5 \%$ of the time, infected animals are detected at the initial test, but no remaining infected animals are left in the herd. In this case, the protocol starts but the herd does not have any infected animals.

To measure the efficacy of test-and-cull protocols, we estimated the probability that bTB is eliminated from the herd as a function of the number of consecutive negative WHT performed in the removal phase. The CDF of the number of removal rests needed to eliminate bTBinfected animals when 1, 5, and 10 bTB-infected animals were introduced into our simulated herd is shown in Figure 3. Our results show that when 1 bTB-infected animal is introduced in a 1,000-cow herd, 1 negative removal test is expected to eliminate bTB infection $79.5 \%$ of the time. That probability when 5 and 10 infected animals are introduced in the herd decreases to 69.1 and $67.7 \%$, respectively. After 2 consecutive negative WHT, the probabilities of bTB elimination in the herd when 1,5 , and 10 infected animals are introduced are 94.7, 94, and 94.5\%, respectively. Our results support the USDA's elimination procedure for bTB outbreaks of up to $1 \%$ of the herd size, in which, in general, 2 consecutive negative WHT result in about $95 \%$ confidence of bTB elimination from the herd. After 3 consecutive negative WHT, the probability of bTB elimination from the herd is at least $99.1 \%$, for all initial infection levels, increasing to about $99.9 \%$ after 4 consecutive negative WHT. When 2 or more consecutive negative WHT are performed in a bTB-infected herd, the probability of elimination does not seem to differ across the initial bTB prevalence analyzed. By the time a WHT is negative, most bTB-positive animals are already eliminated from the herd by the previous positive WHT, including the initial test to estimate prevalence. The reason a herd may require many consecutive negative WHT is that the sensitivity of the CFT test is not perfect and so a bTB-infected animals may not be detected by the test (false negative).

\section{Number of Days for bTB Detection and Elimination}

Next, we estimated the number of days that it takes to detect a bTB-positive animal and to eliminate the infection after the infection is introduced in the herd,

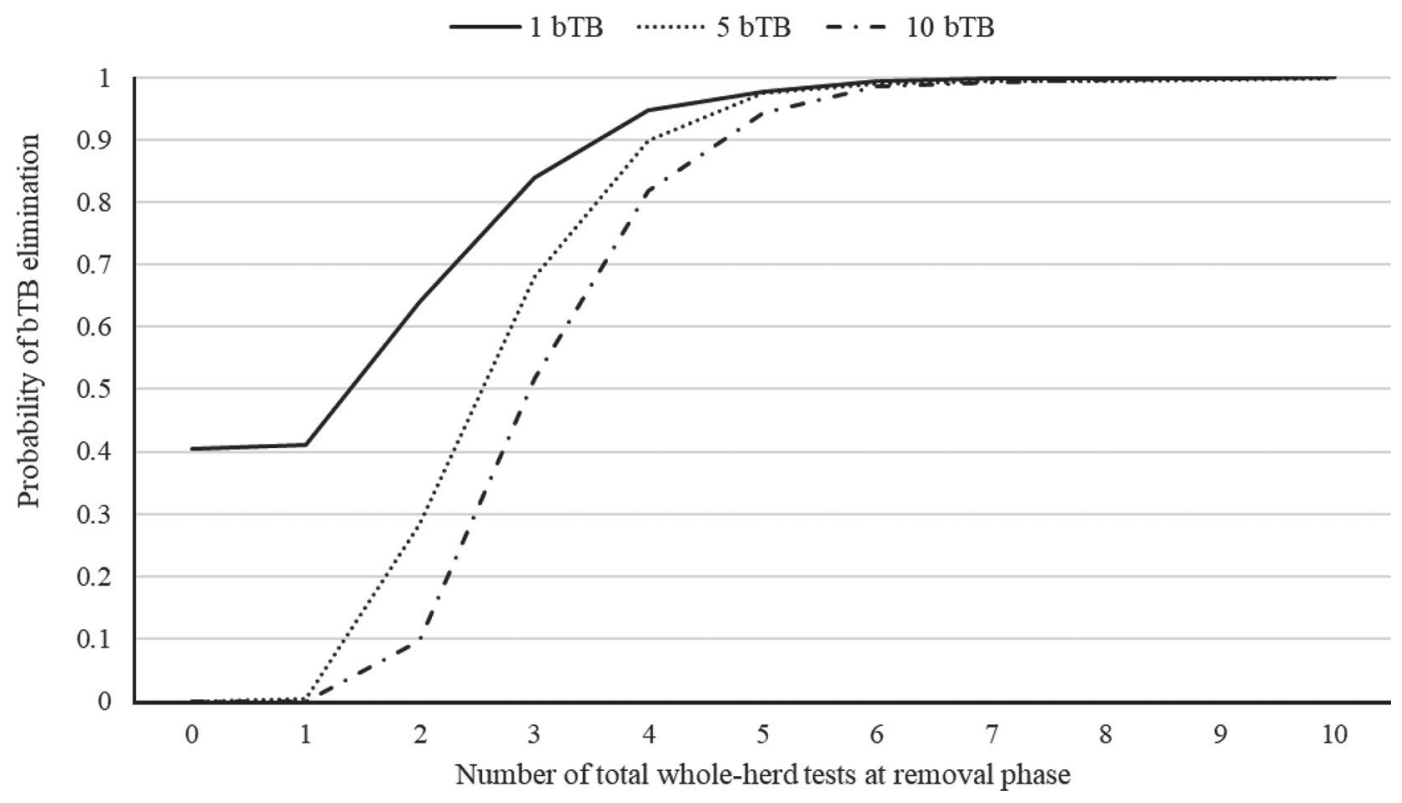

Figure 2. Empirical cumulative distribution of the number of total whole-herd tests in the removal phase needed to eliminate bovine tuberculosis (bTB)-infected animals in a 1,000-cow herd for 3 levels of initial infection, where $1 \mathrm{bTB}, 5 \mathrm{bTB}$, and $10 \mathrm{bTB}$ indicate the initial number of bTB-infected animals introduced into the herd. 


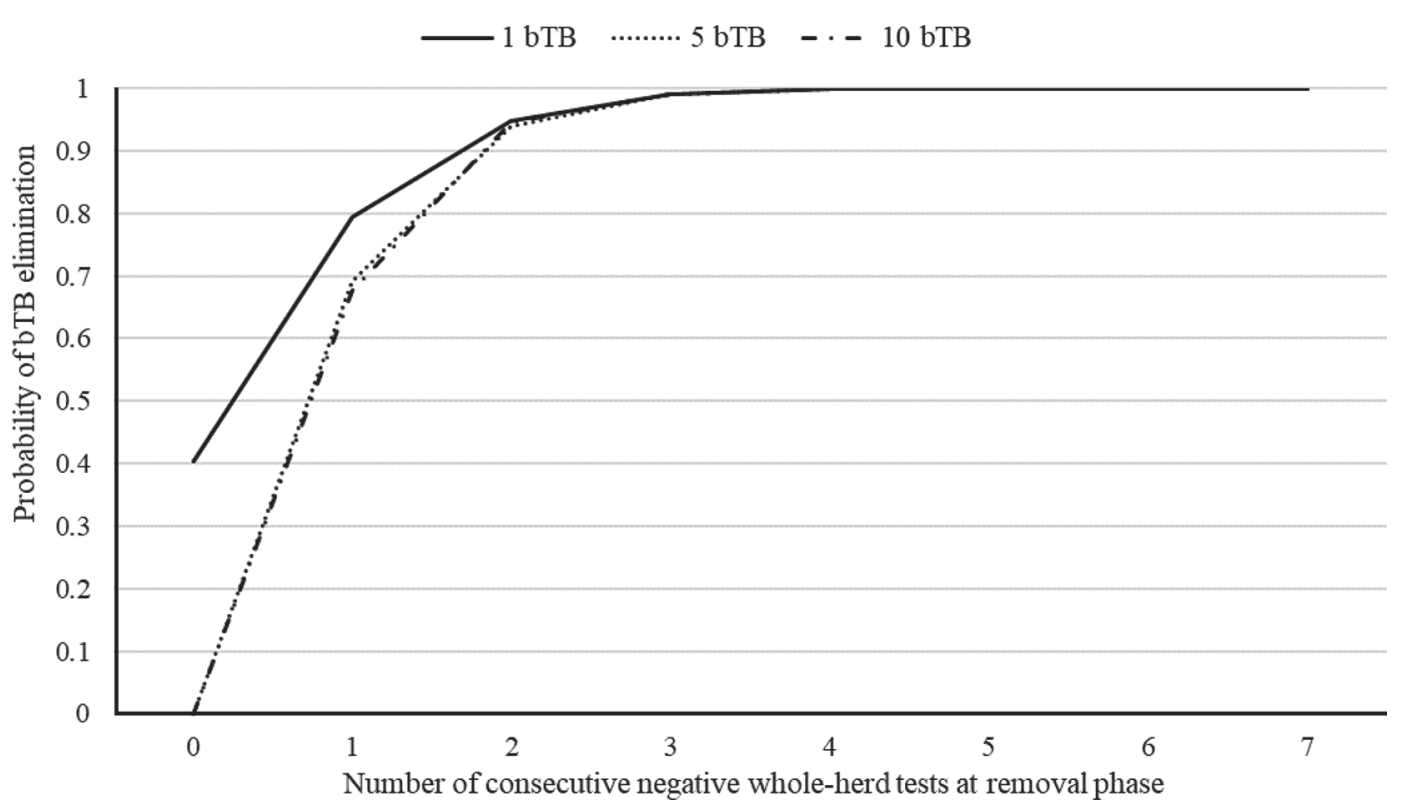

Figure 3. Empirical cumulative distribution of the number of consecutive negative whole-herd tests at removal phase needed to eliminate bovine tuberculosis (bTB)-infected animals in a 1,000-cow herd for 3 levels of initial infection, where $1 \mathrm{bTB}, 5 \mathrm{bTB}$, and $10 \mathrm{bTB}$ indicate the initial number of bTB-infected animals introduced into the herd.

as well as the number of total infected animals remaining in the herd at time of detection. These results are shown in Table 4.

On average, it takes $915 \mathrm{~d}$ to detect a bTB-infected animal after 1 infected animal is introduced into the herd. Detection occurs in the initial test, before the protocol is implemented. After detection, it takes about $202 \mathrm{~d}$ to eliminate bTB-infected animals from the herd. As the number of initial infected animals increases, the average days to detection and its standard deviation decreases. For instance, it takes, on average, $364 \mathrm{~d}$ to detect an infected animal if 5 bTB-infected animals are introduced in the herd, and $288 \mathrm{~d}$ to detect the infection if $10 \mathrm{bTB}$-infected animals are introduced in the herd; the standard deviations are 153 and 85, respectively. The days needed to eliminate a bTB outbreak from the herd increases as the number of initial infected animals increase, from $202 \mathrm{~d}$ for 1 initial infected animal, to 204 and $234 \mathrm{~d}$ for 5 and 10 initial infected animals, respec- tively. A larger number of infected cows entering the herd increases the chance that the infection in the herd is detected earlier. Because the total number of infected animals is greater (the initial infected animals as well as the secondarily infected animals), the probability of slaughterhouse detection increases. When 1 infected animal is introduced in the herd, it is expected that about 4 (4.28) additional infected animals exist in the herd by the time of detection, assuming the introduced animal is detected first. This number increases to 6 and 10 when the number of initial infected animals is 5 and 10 , respectively.

\section{bTB Prevalence Estimation}

The number of true bTB-infected animals remaining in the herd estimated from the results of the initial test are shown next. For the case of 1 infected animal introduced into the herd, the conditional probability

Table 4. Time to detect and eliminate bovine tuberculosis (bTB) in the herd after infected animals are introduced, days taken to eliminate bTB-infected animals from the herd after the protocol is implemented, and number of total bTB-infected animals remaining in the herd at time of detection

\begin{tabular}{lcccc}
\hline $\begin{array}{l}\text { Initial infected } \\
\text { animals }\end{array}$ & $\begin{array}{c}\text { Time to } \\
\text { detection, d }\end{array}$ & $\begin{array}{c}\text { Time to } \\
\text { elimination, d }\end{array}$ & $\begin{array}{c}\text { Time to eliminate } \\
\text { bTB after } \\
\text { detection, d }\end{array}$ & $\begin{array}{c}\text { Total infected animals } \\
\text { remaining in the herd } \\
\text { at time of detection }\end{array}$ \\
\hline 1 & $915(451)$ & $1,117(478)$ & $202(136)$ & $4.28(3.53)$ \\
5 & $364(153)$ & $568(203)$ & $204(112)$ & $5.58(2.65)$ \\
10 & $288(85)$ & $522(148)$ & $234(118)$ & $10.47(3.14)$ \\
\hline
\end{tabular}

${ }^{1}$ Results are for each initial infected number of animals; SD in parentheses. 
that no bTB-infected animals remain in the herd after a negative initial test is $96.6 \%$. When the initial test is positive, regardless of the number of test positives, the probability of having at least 1 infected animal remaining in the herd is $65.5 \%$. For the other initial infection levels analyzed, a positive initial test result indicates almost with certainty that infected animals remain in the herd.

The number of bTB prevalence in the herd depends on the number of bTB positives at the initial test. When 1 bTB-infected animal is introduced into the herd and the initial test results in 1 positive animal, $57.7 \%$ of the time no infected animals will be left in the herd. This probability decreases to $13.6 \%$ when 2 positive animals are found in the initial test. Figure 4 shows the empirical cumulative distribution function of the number of infected animals remaining in the herd after 1 infected animal is introduced in the herd when 1,3 , and 5 positive animals are found in the initial test.

From the results in Figure 4, the probability that 5 or fewer bTB-infected animals are left in the herd after 1 positive initial test result, and after the introduction of 1 infected animal into the herd, is $99.5 \%$. The same probabilities under 3 and 5 positives are 76 and $26.3 \%$, respectively.

A similar pattern is observed when 5 and 10 initial infected animals are introduced into the herd. Supplemental Figure S1 (https://doi.org/10.3168/jds.2018 -14990) shows the empirical cumulative distribution function for the case of 3 positive animals detected in the initial test when 1, 5, and 10 initial infected animals are introduced into the herd. The probability of the number of infected animals remaining in the herd, which is dependent on the number of infected animals introduced in the herd, can be estimated from the number of positives in the initial test. If 3 positives are detected at the initial test, there is a $46 \%$ probability that there are at least 4 infected animals remaining in the herd if 5 infected animals were introduced in the herd at the same time. Similarly, if 10 infected animals are introduced in the herd at the same time, there will be a $58 \%$ probability that at least 8 infected animals remain in the herd.

We also tested whether the number of detected animals in the initial test affects the number of consecutive negative WHT needed to eliminate bTB-infected animals from the herd. We found no evidence that the number of consecutive negative WHT needed to eliminate bTB-infected animals from the herd is related to the number of positives in the initial test for every initial infection level analyzed. The number of total WHT is affected by initial prevalence, requiring more total WHT as the initial prevalence increases.

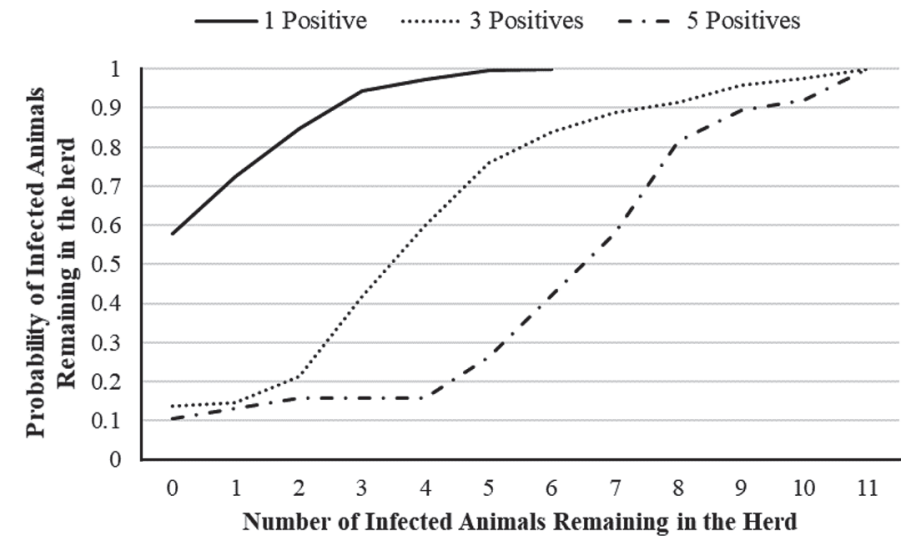

Figure 4. Empirical cumulative distribution of the number of bovine tuberculosis (bTB)-infected animals remaining in the herd after 1 infected animal is introduced in the herd, where 1,3 , and 5 positives indicate the number of bTB-positive animals detected in the initial test.

\section{Reproductive Number}

The estimated $R_{0}$ through time, and the $95 \% \mathrm{CI}$, is shown in Figure 5. One year after the bTB-infected animal is introduced in the herd, the $R_{0}$ estimate is 0.557 (95\% CI: 0.489 to 0.625 ), indicating that, on average, at the end of year 1 , the number of newly infected animals is 0.5 . At yr 2 , the $R_{0}$ is $>1$ (1.554), including the confidence interval (1.419 to 1.689 ), and $R_{0}$ reaches a plateau in yr 10 . The mean $R_{0}$ is 4.715 (95\% CI: 4.343 to 5.087). Our estimate is higher than the estimate of Smith et al. (2013a) at 4.13, but similar to the 4.9 estimate of Conlan et al. (2012). An $R_{0}$ estimate $>1$ indicates that the bTB infection will continue to spread in the population in the absence of a control strategy.

The effective reproduction number, $R_{\mathrm{e}}$, was estimated for the 3 levels of initial infection and under the USDA bTB elimination protocol. The mean $R_{\mathrm{e}}$ estimates and

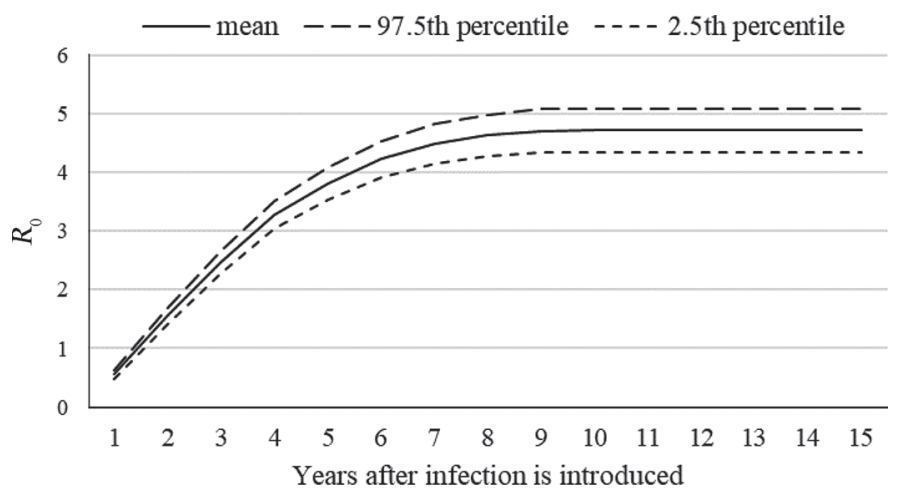

Figure 5. Dynamics of basic reproduction number, $R_{0}$, after infection is introduced in the herd. 
Table 5. Sensitivity analysis of the bovine tuberculosis (bTB) transmission rate on the herd's net present value (\$; SD in parentheses) for different numbers of initial bTB-infected animals following the USDA bTB elimination protocol for transmission rates deviating by $20 \%$ [ $\beta$ Low (2.21) and $\beta$ High (3.31)]

\begin{tabular}{lcc}
\hline \multirow{2}{*}{$\begin{array}{l}\text { Initial infected } \\
\text { animals }\end{array}$} & \multicolumn{2}{c}{ Transmission rate } \\
\cline { 2 - 3 } & BLow & $\beta$ High \\
\hline 1 & $5,639,091$ & $5,568,689$ \\
& $(707,090)$ & $(765,537)$ \\
5 & $4,860,459$ & $4,763,144$ \\
& $(444,396)$ & $(477,258)$ \\
10 & $4,597,299$ & $4,524,947$ \\
& $(437,321)$ & $(453,040)$ \\
\hline
\end{tabular}

95\% CI (in parentheses) for 1, 5, and 10 introduced bTB animals are $0.417(0.395,0.439), 0.312(0.298,0.325)$, and $0.281(0.270,0.291)$, respectively. All values of $R_{\mathrm{e}}$ are $<1$, indicating that the infection will eventually be controlled under the protocol. The $R_{\mathrm{e}}$ value decreases as the number of initial infected animals increases. From Table 4, we can see that the time to detection decreases with the number of initial infected animals. This causes the protocol to begin earlier and thus detection and culling of infected animals occurs before they can infect other animals. Any infected animal that is culled before infecting another animal has an $R_{\mathrm{e}}$ of zero, thereby lowering the $R_{\mathrm{e}}$ average.

\section{Sensitivity Analysis}

We ran a set of simulations where the bTB transmission rate, $\beta$, was increased and decreased by $20 \%$, resulting in values of 2.21 and 3.31 ( $\beta$ Low and $\beta$ High, respectively). Table 5 shows the NPV of the modified transmission rates.

For each initial infection level, the herd's NPV was lower for the higher $(+20 \%)$ transmission rate and higher for the lower $(-20 \%)$ transmission rate. When the transmission rate is higher, 1 infected animal can infect more animals and the number of total WHT needed to eliminate bTB-infected animals from the herd may increase. The time to detection, days to eliminate bTBinfected animals, and total infected animals remaining in the herd after detection for $\beta$ Low and $\beta$ High are shown in Table 6.

For each initial infection level, the number of days to detection was greater for $\beta$ Low than for $\beta$ High. However, as the number of initial infected animals increased (from 1 and 5 to 10) this difference became smaller. The number of days to detection in the case of 10 initial infected animals were 290, 288, and 288 for transmission values of $\beta$ Low, $\beta$, and $\beta$ High, respectively. The number of days needed to eliminate bTB-infected animals from the herd and the number of total infected animals remaining in the herd after detection were larger for the higher $\beta$ value.

The estimates of $R_{\mathrm{e}}$ did not differ statistically for 1 initially infected animal between $\beta, \beta$ Low, and $\beta$ High. The values between $\beta$ Low and $\beta$ High were statistically different for initial infection levels of $5(0.298,0.360)$ and $10(0.247,0.320)$ animals, respectively. For the 10 initially infected animals case, all $R_{\mathrm{e}}$ estimates were statistically different from each other at the $95 \%$ level. The lower transmission rate resulted in a smaller $R_{\mathrm{e}}$ value. For instance, for 1 initial infected animal, the $R_{\mathrm{e}}$ was 0.393 and 0.421 for $\beta$ Low and $\beta$ High, respectively.

The effectiveness of the USDA protocol is not affected meaningfully by changes in the transmission rate. The probabilities of bTB elimination in the herd after 2 consecutive negative WHT, for initial infection levels of 1,5 , and 10 animals, respectively, were $96.6,92.5$, and 93.2\% for $\beta$ Low and 96.6, 94.4, and $91.7 \%$ for $\beta$ High.

Next, we estimated the efficiency of the protocol when we included bTB test parameters that were more conservative than those used by the USDA. The conservative parameter values are shown in Table 7 and are obtained from literature that better reflects the bTB situation in the United States; however, these results can be considered the lower limit of test parameters.

The probability of bTB elimination after 2 consecutive negative WHT under conservative test parameters was estimated at $89.7,81.1$, and $81.8 \%$ for initial infection

Table 6. Sensitivity analysis of time to detect and eliminate bovine tuberculosis (bTB) in the herd after infected animals are introduced, days to eliminate bTB-infected animals from the herd after the protocol is implemented, and number of total bTB-infected animals remaining in the herd at time of detection ${ }^{1}$

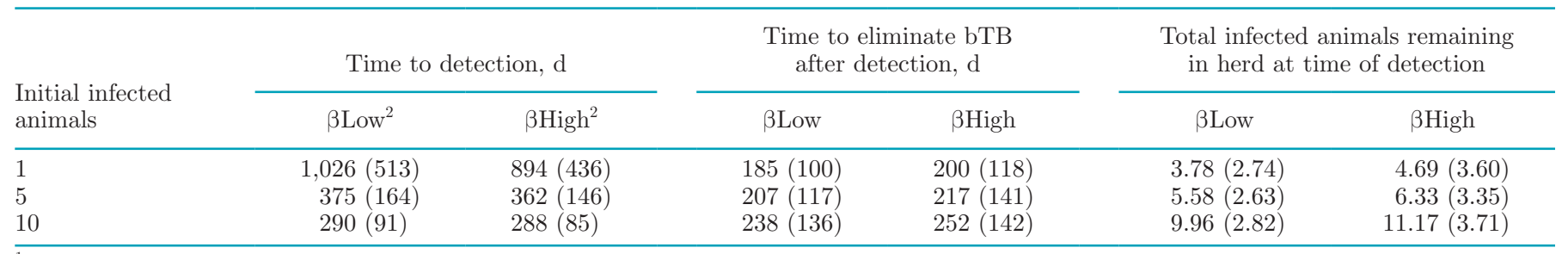

${ }^{1}$ Results are for each initial infected number of animals; SD in parentheses.

${ }^{2} \beta$ Low (2.21) and $\beta$ High (3.31) refer to transmission rates deviating by $20 \%$ from the reference value. 
Table 7. Conservative test sensitivities and specificities [mode, median (95\% CI)] and prior distribution ( $\beta$ ) used in the sensitivity analysis of the USDA bovine tuberculosis (bTB) elimination protocol

\begin{tabular}{|c|c|c|c|c|}
\hline Test $^{1}$ & Sensitivity & Reference & Specificity $^{2}$ & Reference \\
\hline Necropsy & $\begin{array}{l}0.87,0.55(0.10,0.87) \\
\beta(3,2.52)\end{array}$ & Willeberg et al. (2018) & $\begin{array}{l}0.70,0.70(0.59,0.80) \\
\beta(48,21)\end{array}$ & Strain et al. (2011) \\
\hline Histology & $\begin{array}{l}0.75,0.61(0.85,0.99) \\
\beta(2.10,1.37)\end{array}$ & Nuñez-Garcia et al. (2018) & $\begin{array}{l}0.81,0.78(0.57,0.93) \\
\beta(14,4)\end{array}$ & $\begin{array}{l}\text { Bermudez et al. (2010); Varello } \\
\text { et al. (2008) }\end{array}$ \\
\hline
\end{tabular}

${ }^{1} \mathrm{CFT}=$ caudal fold tuberculin; CCT $=$ comparative cervical tuberculin. The parameter values of CCT test are estimated for series testing (CFT-CCT).

${ }^{2}$ Specificities of CFT, necropsy, and histology are taken from the USDA bTB protocol.

levels of 1,5 , and 10 animals, respectively. These probabilities increase to $97.8,94.5$, and $94.5 \%$, respectively, after 3 consecutive negative WHT. The total number of WHT needed to eliminate bTB-infected animals in the herd for all initial levels of infected animals is 6 , with confidence of at least $95 \%$. This is similar to the value of using the USDA's test parameters.

The estimates of $R_{\mathrm{e}}$ under the conservative bTB test parameters were higher than those following the USDA test parameters. The $R_{\mathrm{e}}$ values for 1,5 , and 10 initial infected animals were $0.436,0.417$, and 0.328 , respectively.

\section{General Discussion}

This paper evaluated the current USDA bTB elimination protocol in a 1,000-cow US commercial dairy herd, when the initial numbers of bTB-infected heifers were 1,5 , and 10 . We assumed a closed dairy herd with no possibility of wildlife transmission. The bTB-infected heifers were classified as occult; that is, animals that are infected with $M$. bovis but did not show signs of the infection, were not detectable by bTB tests, and cannot yet infect other healthy animals. Occult animals become reactive animals, which can be detected by testing but cannot infect other animals. Reactive animals become infectious animals, which can be detected by testing and can infect other animals.

Our results indicated that the USDA bTB elimination protocol applied in a closed dairy herd operation is effective in eradicating the infection while minimizing costs. We tested the assumption that after 2 consecutive negative WHT, there is a $95 \%$ probability of eliminating bTB-infected animals in the herd. These results are based on the USDA bTB test parameter estimates (sensitivity and specificity). These test parameter values are conservative compared with the relevant literature. In general, the mean values of the test parameters used by the USDA in evaluating its bTB elimination protocol are lower than a pooled average from the literature relevant to the US situation. However, we tested the model using even more conservative test parameter values, relevant to the US situation, found in the literature. Under these lower test parameter values, it would take 3 consecutive negative WHT to eliminate bTB-infected animals with a confidence level of $95 \%$ for all initial infection levels simulated $(0.1,0.5$, and $1 \%$ ). These test parameter values are very low and may not reflect expected test parameter values. The $R_{\mathrm{e}}$ of performing the elimination protocol for any infection level analyzed was $<0.42$, indicating that the protocol is effective in controlling the spread of the infection.

Applying the USDA bTB elimination protocol to a healthy herd of 1,000 cows with initial bTB infection levels of 1 and $0.1 \%$ resulted in 32 to $21 \%$ lower NPV compared with a healthy herd, respectively.

Some limitations of this study include the assumptions of a closed herd, no wildlife transmission of bTB, and effective pasteurization of calf milk. Although bTB is rare in most of the United States, the possibility of infection from the introduction of infected replacement animals and from wildlife reservoirs may produce different results from those presented in this paper. Ineffective pasteurization of calf milk can increase the bTB transmission rate to calves. The time and cost associated with elimination of bTB-infected animals from the herd may increase if these limitations are considered.

\section{ACKNOWLEDGMENTS}

The authors gratefully acknowledge funding provided by the National Institute of Food and Agriculture of the United States Department of Agriculture (Washington, DC) through NIFA Award No. 2014-67015- 2240. The authors thank Michael Carter, Kathleen Orloski, and the 2 anonymous referees for helpful comments. 


\section{REFERENCES}

Al-Mamun, M. A., R. L. Smith, Y. H. Schukken, and Y. T. Grohn. 2016. Modeling of Mycobacterium avium ssp. paratuberculosis dynamics in a dairy herd: An individual based approach. J. Theor. Biol. 408:105-117. https://doi.org/10.1016/j.jtbi.2016.08.014.

Álvarez, J., J. Bezos, M. L. de la Cruz, C. Casal, B. Romero, L. Domínguez, and A. Pérez., 2014. Bovine tuberculosis: Within-herd transmission models to support and direct the decision-making process. Res. Vet. Sci. 97(Suppl.):S61-S68. https://doi.org/10 $.1016 /$ j.rvsc.2014.04.009.

Asseged, B., Z. Woldesenbet, E. Yimer, and E. Lemma. 2004. Evaluation of abbatoir inspection for the diagnosis of Mycobacterium bovis infection in cattle at Addis Ababa abbatoir. Trop. Anim. Health Prod. 36:537-546.

Barlow, N. D., J. M. Kean, G. Hickling, P. G. Livingstone, and A. B. Robson. 1997. A simulation model for the spread of bovine tuberculosis within New Zealand cattle herds. Prev. Vet. Med. 32:57-75. https://doi.org/10.1016/S0167-5877(97)00002-0.

Bermudez, H. R., E. T. Renteria, B. G. Medina, S. Hori-Oshim, A. De la Mora-Valle, V. G. Lopez, W. L. Yu, G. R. Pio, J. C. Herrera, C. Pujol, and K. Nielsen. 2010. Correlation between histopathological, bacteriological and PCR diagnosis of bovine tuberculosis. J. Anim. Vet. Adv. 9:2082-2084. https://doi.org/10.3923/javaa.2010 .2082 .2084

Bruning-Fann, C. S., S. Robbe-Austerman, J. B. Kaneene, B. V. Thomsen, J. D. Tilden Jr., J. S. Ray, R. W. Smith, S. D. Fitzgerald, S. R. Bolin, D. J. O'Brien, and T. P. Mullaney. 2017. Use of whole-genome sequencing and evaluation of the apparent sensitivity and specificity of antemortem tuberculosis tests in the investigation of an unusual outbreak of Mycobacterium bovis infection in a Michigan dairy herd. J. Am. Vet. Med. Assoc. 251:206-216.

Buhr, B. K., McKeever, and K. Adachi. 2009. Economic impact of bovine tuberculosis on Minnesota's cattle and beef sector. Page 20 in Michigan Bovine Tuberculosis Bibliography and Database, St. Paul, MN

Conlan, A. J. K., T. J. McKinley, K. Karolemeas, E. B. Pollock, A. V. Goodchild, A. P. Mitchell, C. P. Birch, R. S. Clifton-Hadley, and J. L. N. Wood. 2012. Estimating the hidden burden of bovine tuberculosis in Great Britain. PLOS Comput. Biol. 8:e1002730. https://doi.org/10.1371/journal.pcbi.1002730.

de la Rua-Domenech, R., A. T. Goodchild, H. M. Vordermeier, R. G. Hewinson, K. H. Christiansen, and R. S. Clifton-Hadley. 2006. Ante mortem diagnosis of tuberculosis in cattle: A review of the tuberculin tests, $\gamma$-interferon assay and other ancillary diagnostic techniques. Res. Vet. Sci. 81:190-210.

Downs, S. H., J. E. Parry, P. A. Upton, J. M. Broughan, A. V. Goodchild, J. Nuñez-Garcia, and R. S. Clifton-Hadley. 2018. Methodology and preliminary results of a systematic literature review of ante-mortem and post-mortem diagnostic tests for bovine tuberculosis. Prev. Vet. Med. 153:117-126. https://doi.org/10.1016/j .prevetmed.2017.11.004.

Dressler, J. B., R. L. Smith, L. W. Tauer, Y. H. Schukken, and Y. T. Grohn. 2010. Economic analysis of the cross-reactivity of Johne's disease vaccination with tuberculosis in dairy cattle. Am. J. Agric. Econ. 92:1446-1455. https://doi.org/10.1093/ajae/aaq075.

European Food Safety Authority (EFSA). 2009. Trends and sources of zoonoses and zoonotic agents in the European Union in 2007. EFSA, Parma, Italy.

Farnham, M. W., B. Norby, T. J. Goldsmith, and S. J. Wells. 2012. Meta-analysis of field studies on bovine tuberculosis skin tests in United States cattle herds. Prev. Vet. Med. 103:234-242. https:// doi.org/10.1016/j.prevetmed.2011.08.009.

Fischer, E. A. J., H. J. W. Van Roermund, L. Hemerik, M. A. P. M. Van Asseldonk, and M. C. M. De Jong. 2005. Evaluation of surveillance strategies for bovine tuberculosis (Mycobacterium bovis) using an individual based epidemiological model. Prev. Vet. Med. 67:283-301. https://doi.org/10.1016/j.prevetmed.2004.12.002.

Kaniyamattam, K., M. A. Elzo, J. B. Cole, and A. De Vries. 2016. Stochastic dynamic simulation modeling including multitrait genetics to estimate genetic, technical, and financial consequences of dairy farm reproduction and selection strategies. J. Dairy Sci. 99:8187-8202. https://doi.org/10.3168/jds.2016-11136.

Kao, R. R., M. G. Roberts, and T. J. Ryan. 1997. A model of bovine tuberculosis control in domesticated cattle herds. Proc. Biol. Sci. 264:1069-1076. https://doi.org/10.1098/rspb.1997.0148.

Karszes, J. 2014. Dairy Replacement Programs: Costs and Analysis 3rd Quarter 2012. Extension Bulletin 2014-02. Department of Animal Science and Charles H. Dyson School of Applied Economics and Management, Cornell University, Ithaca, NY.

Korver, S., J. A. M. van Arendonk, and W. J. Koops. 1985. A function for live-weight change between two calvings in dairy cattle. Anim. Prod. 40:233-241. https://doi.org/10.1017/S0003356100025332.

Lahuerta-Marin, A., M. G. Milne, J. Mcnair, R. A. Skuce, S. H. Mcbride, F. D. Menzies, S. J. W. Mcdowell, A. W. Byrne, I. G. Handel, C. De, and B. M. Bronsvoort. 2018. Bayesian latent class estimation of sensitivity and specificity parameters of diagnostic tests for bovine tuberculosis in chronically infected herds in Northern Ireland. Vet. J. 238:15-21. https://doi.org/10.1016/j.tvjl.2018 .04 .019 .

Livingstone, P. G., T. J. Ryan, N. G. Hancox, K. B. Crews, M. A. J. Bosson, G. J. E. Knowles, and W. McCook. 2006. Regionalisation: A strategy that will assist with bovine tuberculosis control and facilitate trade. Vet. Microbiol. 112:291-301. https://doi.org/10 .1016/j.vetmic.2005.11.016.

Mellado, M., D. Reséndiz, A. M. Martínez, M. A. de Santiago, F. G. Véliz, and J. E. García. 2015. Milk yield and reproductive performance of Holstein cows testing positive for bovine tuberculosis. Trop. Anim. Health Prod. 47:1061-1066. https://doi.org/10.1007/ s11250-015-0828-1.

Mitchell, R. M., R. H. Whitlock, S. M. Stehman, A. Benedictus, P. P. Chapagain, Y. T. Grohn, and Y. H. Schukken. 2008. Simulation modeling to evaluate the persistence of Mycobacterium avium ssp. paratuberculosis (MAP) on commercial dairy farms in the United States. Prev. Vet. Med. 83:360-380. https://doi.org/10.1016/j .prevetmed.2007.09.006.

More, S. J., and M. Good. 2015. Understanding and managing bTB risk: Perspectives from Ireland. Vet. Microbiol. 176:209-218. https: //doi.org/10.1016/j.vetmic.2015.01.026.

More, S. J., B. Radunz, and R. J. Glanville. 2015. Lessons learned during the successful eradication of bovine tuberculosis from Australia. Vet. Rec. 177:224-232. https://doi.org/10.1136/vr.103163.

Naugle, A. N., M. Schoenbaum, C. W. Hench, O. L. Henderson, and J. Shere. 2014. Bovine tuberculosis eradication in the United States. Page 238 in Zoonotic Tuberculosis: Mycobacterium bovis and Other Pathogenic Mycobacteria. 3rd ed. C. O. Thoen, J. H. Steele, and J. B. Kaneen, ed. John Wiley and Sons Inc., New York, NY.

Norby, B. 2003. Performance of bovine tuberculin skin tests and factors affecting the proportion of false positive skin test results in Michigan. Michigan State University, East Lansing, MI

Norby, B., P. C. Bartlett, S. D. Fitzgerald, L. M. Granger, C. S. Bruning-Fann, D. L. Whipple, and J. B. Payeur. 2004. The sensitivity of gross necropsy, caudal fold and comparative cervical tests for the diagnosis of bovine tuberculosis. J. Vet. Diagn. Invest. 16:126131. https://doi.org/10.1177/104063870401600206.

NRC. 2001. Nutrient Requirements of Dairy Cattle. 7th rev. ed. National Academies Press, Washington, DC.

Nuñez-Garcia, J., S. H. Downs, J. E. Parry, D. A. Abernethy, J. M. Broughan, A. R. Cameron, and M. Greiner. 2018. Meta-analyses of the sensitivity and specificity of ante-mortem and post-mortem diagnostic tests for bovine tuberculosis in the UK and Ireland. Prev. Vet. Med. 153:94-107. https://doi.org/10.1016/j.prevetmed .2017.02.017.

O'Hare, A., R. J. Orton, P. R. Bessell, and R. R. Kao. 2014. Estimating epidemiological parameters for bovine tuberculosis in British cattle using a Bayesian partial-likelihood approach. Proc. R. Soc. B Biol. Sci. 281:20140248. https://doi.org/10.1098/rspb.2014 .0248 .

Perez, A. M., M. P. Ward, A. Charmandarián, and V. Ritacco. 2002. Simulation model of within-herd transmission of bovine tuberculosis in Argentine dairy herds. Prev. Vet. Med. 54:361-372. https:// doi.org/10.1016/S0167-5877(02)00043-0. 
Radunz, B. 2006. Surveillance and risk management during the latter stages of eradication: Experiences from Australia. Vet. Microbiol. 112:283-290. https://doi.org/10.1016/j.vetmic.2005.11.017.

Ryan, T. J., P. G. Livingstone, D. S. L. Ramsey, G. W. de Lisle, G. Nugent, D. M. Collins, and B. M. Buddle. 2006. Advances in understanding disease epidemiology and implications for control and eradication of tuberculosis in livestock: The experience from New Zealand. Vet. Microbiol. 112:211-219. https://doi.org/10.1016/j .vetmic.2005.11.025.

Schiller, I., W. R. Waters, H. M. Vordermeier, T. Jemmi, M. Welsh, N. Keck, A. Whelan, E. Gormley, M. L. Boschiroli, J. L. Moyen, C. Vela, M. Cagiola, B. M. Buddle, M. Palmer, T. Thacker, and B. Oesch. 2011. Bovine tuberculosis in Europe from the perspective of an officially tuberculosis free country: Trade, surveillance and diagnostics. Vet. Microbiol. 151:153-159. https://doi.org/10.1016/ j.vetmic.2011.02.039.

Silva Del Río, N. S., S. Stewart, P. Rapnicki, Y. M. Chang, and P. M. Fricke. 2007. An observational analysis of twin births, calf sex ratio, and calf mortality in Holstein dairy cattle. J. Dairy Sci. 90:1255-1264. https://doi.org/10.3168/JDS.S0022-0302(07)71614 -4 .

Smith, R. L., Y. H. Schukken, Z. Lu, R. M. Mitchell, and Y. T. Grohn. 2013a. Development of a model to simulate infection dynamics of Mycobacterium bovis in cattle herds in the United States. J. Am. Vet. Med. Assoc. 243:411-423. https://doi.org/10.2460/javma.243 .3.411.

Smith, R. L., L. W. Tauer, M. W. Sanderson, and Y. T. Grohn. 2014. Minimum cost to control bovine tuberculosis in cow-calf herds. Prev. Vet. Med. 115:18-28. https://doi.org/10.1016/j.prevetmed 2014.03.014

Smith, R. L., L. W. Tauer, Y. H. Schukken, Z. Lu, and Y. T. Grohn. 2013b. Minimization of bovine tuberculosis control costs in US dairy herds. Prev. Vet. Med. 112:266-275. https://doi.org/10 .1016/j.prevetmed.2013.07.014.

Strain, S. A. J., J. Mcnair, and S. W. J. Mcdowell. 2011. Bovine tuberculosis: A review of diagnostic tests for $M$. bovis infection in badgers. Accessed Oct. 5, 2018. https://pdfs.semanticscholar.org/ 350a/f4a5eea6bd351dca790b0416c817b583c007.pdf.

USAHA. 2004. Report of the Committee on Tuberculosis, Accessed Oct. 5, 2018. http://portals5.gomembers.com/Portals/6/Reports/ 2004/report-tb- 2004.pdf.

USDA-APHIS (Animal and Plant Health Inspective Service). 2005. Bovine tuberculosis eradication uniform methods and rules, APHIS 91-4, 91-45-011. Accessed May 3, 2017. http://www.aphis.usda .gov/animal_health/animal_diseases/tuberculosis/downloads/tb -umr.pdf.

USDA-APHIS (Animal and Plant Health Inspective Service). 2009. New approach for managing bovine tuberculosis: Veterinary
Services' proposed action plan. Accessed Jul. 18, 2017. http:// digitalcommons.unl.edu/cgi/viewcontent.cgi? article $=1091 \&$ context $=$ michbovinetb.

USDA-APHIS (Animal and Plant Health Inspective Service). 2017. Status of current eradication programs. Accessed Jul. 18, 2017. https://www.aphis.usda.gov/aphis/ourfocus/animalhealth/animal -disease-information/ct_status_of_eradication_programs.

USDA-APHIS-VS. (Animal and Plant Health Inspective Service, Veterinary Services). n.d. Memorandum 552.47, Procedures for Managing Bovine Tuberculosis-Affected Cattle Herds.

USDA-APHIS-VS. (Animal and Plant Health Inspective Service, Veterinary Services). 2010. Questions and Answers: Bovine Tuberculosis Federal Order, April, 2010. Accessed Sep. 15, 2018. https: //www.aphis.usda.gov/publications/animal_health/content/ printable_version/faq_bovine_tb_fed_order.pdf.

USDA-ERS (Economic Research Service). 2015. National milk cost of production. Accessed Jul. 18, 2017. https://www.ers.usda.gov/ data-products/milk-cost-of-production-estimates.aspx.

USDA-NASS (National Agricultural Statistics Service). 2017. Agricultural prices. Accessed Sep. 20, 2017. http://usda.mannlib.cornell .edu/usda/nass/AgriPric//2010s/2017/AgriPric-03-30-2017.pdf.

Varello, K., M. Pezzolato, D. Mascarino, F. Ingravalle, M. Caramelli, and E. Bozzetta. 2008. Comparison of histologic techniques for the diagnosis of bovine tuberculosis in the framework of eradication programs. J. Vet. Diagn. Invest. 20:164-169. https://doi.org/10 $.1177 / 104063870802000204$

Verteramo Chiu, L. J., L. W. Tauer, M. A. Al-Mamun, K. Kaniyamattam, R. L. Smith, and Y. T. Grohn. 2018. An agent-based model evaluation of economic control strategies for paratuberculosis in a dairy herd. J. Dairy Sci. 101:6443-6454. https://doi.org/10.3168/ jds.2017-13175.

Whipple, D. L., C. A. Bolin, A. J. Davis, J. L. Jarnagin, D. C. Johnson, R. S. Nabors, J. B. Payeur, D. A. Saari, A. J. Wilson, and M. M. Wolf. 1995. Comparison of sensitivity of the caudal fold skin test and commercial gamma-interferon assay for diagnosis of bovine tuberculosis. Am. J. Vet. Res. 56:415-419.

Willeberg, P. W., C. G. McAloon, E. Houtsma, I. Higgins, T. A. Clegg, and S. J. More. 2018. The herd-level sensitivity of abattoir surveillance for bovine tuberculosis: Simulating the effects of current and potentially modified meat inspection procedures in Irish cattle. Front. Vet. Sci. 5:82. https://doi.org/10.3389/fvets.2018.00082.

Wood, P. R., L. A. Corner, J. S. Rothel, J. L. Ripper, T. Fifis, B. S McCormick, B. Francis, L. Melville, K. Small, K. De Witte, J. Tolson, T. J. Ryan, G. W. de Lisle, J. C. Cox, and S. L. Jones. 1992. A field evaluation of serological and cellular diagnostic tests for bovine tuberculosis. Vet. Microbiol. 31:71-79. https://doi.org/ 10.1016/0378-1135(92)90142-G. 